\title{
REVISÃO DE MÉTODOS DE CÁLCULO DA DIVERGÊNCIA E UMA APLICAÇÃO USANDO O MÉTODO CINEMÁTICO PARA DETERMINAÇÃO DE MOVIMENTOS VERTICAIS NA ATMOSFERA
}

\author{
HELBER BARROS GOMES ${ }^{1}$ e MARCO ANTONIO MARINGOLO LEMES ${ }^{2}$
}

${ }^{1,2}$ Universidade Federal de Alagoas (UFAL), Departamento de Meteorologia.

Campus A. C. Simões, BR 104, Norte, km 97, Tabuleiro dos Martins, Maceió, Alagoas, CEP 57072-970.

E-mail: ${ }^{1}$ helber@model.iag.usp.br e ${ }^{2}$ lemes_marco@hotmail.com

Recebido Agosto 2005 - Aceito Setembro 2006

\begin{abstract}
RESUMO
Esse trabalho revisa três métodos numéricos distintos de se calcular as grandezas cinemáticas presentes na análise de um campo de vento quase horizontal, a saber, a divergência horizontal, a vorticidade vertical e a deformação, a partir de observações irregularmente espaçadas. A motivação para esse estudo deve-se ao fato do crescente número de experimentos de campo, em especial na América do Sul, quando então observações especiais de radio-sondagem são feitas em horários não sinóticos. Aqui, é considerado a título de ilustração, o conjunto de dados de ar superior obtidos durante o Experimento LBA/TRMM (Large Scale Biosphere-Atmosphere Experiment in Amazônia / Tropical Rainfall Measuring Mission), de 24 de janeiro a 24 de fevereiro de 1999. Os métodos revisados incluem o método da integral (Teoremas do Cálculo Vetorial), o método da matriz (Teorema da decomposição de campos Vetoriais de Cauchy - Stokes) e o método dos mínimos quadrados. Os três métodos foram validados com dados teóricos e produziram os mesmos resultados numéricos (dentro da precisão das máquinas usadas). A divergência horizontal foi escolhida para ser usada como dado de entrada para gerar perfis verticais da componente vertical $\mathrm{w}$ através do método cinemático. Os valores de W da ordem de $10 \mathrm{~cm} \mathrm{~s}^{-1}$ são consistentes com os estimados usando uma análise simples de escala para escalas horizontais da ordem de $100 \mathrm{~km}$. É enfatizado que nenhum problema meteorológico foi considerado como objetivo desse trabalho.
\end{abstract}

Palavras-chave: divergência horizontal; movimento vertical; LBA/TRMM.

\begin{abstract}
REVISION OF METHODS OF CALCULATION OF THE DIVERGENCE AND AN APPLICATION USING THE KINEMATIC METHOD FOR DETERMINATION OF VERTICAL MOTIONS IN THE ATMOSPHERE.

This study revises three different methods of calculating the kinematic variables that appear in the analysis of a quasi horizontal wind field: horizontal divergence, vertical vorticity and deformation using observations at irregularly spaced points. The motivation for this study was the increasing number of field experiments in South America when special upper air observations are made. Some upper air data measured in the Large Scale Biosphere-Atmosphere Experiment in Amazonas / Tropical Rainfall Measuring Mission (LBA / TRMM) during January 24 / February 241999 were used to illustrate the methods. The methods are the formal integral definitions of the kinematic quantities based on the fundamental theorems of Vector Calculus, the matrix method based on the decomposition theorem due to Cauchy e Stokes and, finally the least minimum method. The horizontal divergence was chosen as input data to generate vertical profiles of vertical motion w via the kinematic method. The values of $\mathrm{w}$ or order of $10 \mathrm{~cm} \mathrm{~s}^{-1}$ are consistent with those estimated from an rough scale analysis appropriated for horizontal scales of order of $100 \mathrm{~km}$. It is emphasized that no particular meteorological problem is addressed to.
\end{abstract}

Keywords: horizontal divergence, vertical motion, LBA/ TRMM. 


\section{INTRODUÇÃO}

O campo de movimento vertical tem um papel fundamental nos processos dinâmicos e termodinâmicos que ocorrem na atmosfera, em suas mais variadas escalas espaciais, estendendo-se desde as escalas consideradas pela Micrometeorologia até as escalas sinóticas e planetárias. Participa, por exemplo, dos fluxos turbulentos verticais de momentum, energia, vapor de água e outros gases minoritários (porém radiativa e quimicamente importantes) que essencialmente fazem a comunicação entre a camada limite planetária (no seu sentido mais amplo de incluir diferentes tipos de cobertura de solo e de vegetação) e a atmosfera livre acima. Sob esse aspecto, esses fluxos verticais são essenciais para a compreensão de fenômenos de escalas climáticas como o efeito estufa e o problema do aquecimento global. Essa preocupação está presente nos grandes objetivos de programas internacionais que buscam entender, em particular, o papel da Região Amazônica e outras florestas tropicais, face o crescente quadro de desmatamento por quais passam atualmente.

Já em escalas menores, como as escalas meso- $\beta$ e meso- $\alpha$ de Orlansky (1975), o movimento vertical é uma variável essencial à previsões confiáveis de precipitação dentro dos sistemas sinóticos e de escalas regionais. Mas sua importância não se restringe somente ao processo de saturação e precipitação da água na presença de correntes ascendentes. A instabilidade baroclínica [Charney (1947) e Eady (1949)] é o mecanismo de transporte do excesso de energia que a Terra ganha no cinturão tropical para os pólos e, nesse contexto, o movimento vertical é parte da chamada circulação secundária que se desenvolve nas latitudes médias (em especial, latitude - altura) que mantém a atmosfera dentro de um estado de equilíbrio climatológico. Desenvolvimentos mais recentes que enfocam o papel do movimento vertical em grande escala são os melhoramentos na equação omega da Teoria Quase Geostrófica (Holton, 1992), com a introdução do vetor Q (Hoskins et al., 1978; Trenberth 1978).

As dificuldades básicas que caracterizam os movimentos verticais em grandes escalas são sua pequena magnitude (da ordem de cm. $\mathrm{s}^{-1}$ ) quando comparados aos correspondentes movimentos horizontais (da ordem de $10 \mathrm{~m} . \mathrm{s}^{-1}$ ) e a impossibilidade de serem diretamente observados. Ainda, como os movimentos de grande escala caracterizam-se por uma pequena razão de aspecto, eles são quase hidrostáticos e, portanto determinados diagnosticamente dentro dos modelos de Previsão Numérica de Tempo (PNT).

Historicamente na era anterior a PNT surgiram, em contexto isolado, vários métodos de determinação do movimento vertical em escala sinótica. Entre eles, o método termodinâmico (baseado na Primeira Lei da Termodinâmica), o método da advecção de vorticidade (baseado na equação da vorticidade e até hoje qualitativamente usado na interpretação de cartas sinóticas) e o método cinemático (baseado na equação de conservação de massa e usado dentro dos modelos de PNT, por exemplo no cálculo de sigma ponto, a componente vertical da velocidade em modelos de coordenadas sigma). Relativamente poucos trabalhos específicos sobre movimentos verticais sobre a América do Sul podem ser encontrados, mas Bertolossi (1988) fez um estudo de caráter observacional sobre a aplicação desses métodos, para caracterizar o padrão de movimentos verticais associados a vários sistemas sinóticos atuantes nesse continente.

O método cinemático, apesar do inconveniente de ser extremamente sensível a pequenos erros no campo do vento horizontal $^{1}$ (Holton, 1992), foi usado nesse trabalho, pois utiliza somente informações de vento em um único instante de tempo, Procedimentos padrões para minimizar os erros acumulativos na integração vertical da equação da continuidade (usando o perfil vertical de divergência horizontal), como o esquema de O`Brien (1969) que propôs um ajustamento de segunda ordem que exige a especificação de duas condições de contorno, para a velocidade vertical (velocidade vertical nula na superfície e próximo à tropopausa) podem ser encontrados em Krishnamurty (1986). Essa imposição de natureza matemática não permite, portanto dados de radiossondagem que, por qualquer razão, terminem abaixo da tropopausa.

O presente trabalho foi motivado pela disponibilidade de um conjunto de dados de ar superior obtidos em uma fase do Experimento LBA/TRMM (Large Scale Biosphere-Atmosphere Experiment in Amazônia / Tropical Rainfall Measuring Mission) durante 24 de janeiro a 24 de fevereiro que, no conhecimento dos autores, ainda não havia sido utilizado em um contexto sinótico inerente ao experimento, apesar de constituírem dados meteorológicos bastante onerosos. Um dos poucos trabalhos similares publicados onde foram usados dados de radiossondas irregularmente distribuídos (5 estações) para obtenção do movimento vertical é o de Yanai et al. (1973) cujo propósito era estudar o papel do calor latente e umidade em complexos convectivos nos trópicos.

É importante enfatizar que o presente trabalho teve como objetivo principal implementar e testar três métodos (Métodos da Matriz, Integral e Mínimo Quadrado) de determinação da divergência horizontal (igualmente aplicáveis para a vorticidade e deformação) necessária no método cinemático. Não houve, portanto, nenhuma preocupação em se usar o software desenvolvido a qualquer situação prática no contexto meteorológico, exceto aquele de mostrar o perfil vertical de w.

\footnotetext{
Em se tratando de previsão numérica, esse problema é bastante minimizado na fase de pré-processamento (critérios de controle de qualidade e métodos de iniciação).
} 


\section{MATERIAL E MÉTODOS}

\subsection{Métodos Utilizados}

Esta seção subdivide-se em duas partes, onde a primeira aborda os três métodos e a segunda, considera o método cinemático e a sua implementação numérica para se obter o perfil vertical de movimento vertical. Alguns experimentos de validação usando dados teórico e real são comentados.

\subsubsection{Método da Matriz}

A análise linear de um escoamento quase-horizontal (caso dos movimentos atmosféricos de grande escala) consiste em uma expansão das componentes do vento em série de Taylor em torno de um ponto A (ponto da análise), onde a velocidade é conhecida, com o propósito de se determinar os valores da velocidade em um ponto B qualquer de sua vizinhança. Seguindo os procedimentos em Hess (1979), Bluestein (1992) e Lemes \& Moura (2002), a decomposição de movimento horizontal em translação, rotação e deformação (linear e angular) é expressa pela equação matricial:

$\left(\begin{array}{l}\mathrm{u} \\ \mathrm{v}\end{array}\right)_{\mathrm{B}}=\left(\begin{array}{l}\mathrm{u} \\ \mathrm{v}\end{array}\right)_{\mathrm{A}}+\frac{1}{2}\left[\left(\begin{array}{cc}\cdot & 0 \\ 0 & \cdot\end{array}\right)_{\mathrm{A}}+\left(\begin{array}{cc}0 & \mathrm{q} \\ -\boldsymbol{q} & 0\end{array}\right)_{\mathrm{A}}+\left(\begin{array}{cc}\mathrm{D}_{1} & \mathrm{D}_{2} \\ \mathrm{D}_{2} & -\mathrm{D}_{1}\end{array}\right)_{\mathrm{A}}\right]\left(\begin{array}{l}\mathrm{dx} \\ \mathrm{dy}\end{array}\right)$,

onde $\delta=\mathrm{u}_{\mathrm{x}}+\mathrm{v}_{\mathrm{y}}$ é a divergência horizontal, $\zeta=-\mathrm{u}_{\mathrm{y}}+\mathrm{v}_{\mathrm{x}}$ é a componente vertical da vorticidade e $\mathrm{D}_{1}=\mathrm{u}_{\mathrm{y}}+\mathrm{v}_{\mathrm{x}}, \mathrm{D}_{2}=\mathrm{u}_{\mathrm{x}}-\mathrm{v}_{\mathrm{y}}$ são os dois termos de deformação. O método baseia-se na solução da equação (1) e foi desenvolvido para o caso de somente três observações não colineares para ser usado com dados do LBA / TRMM. O método da matriz fornece simultaneamente os valores dessas quatro grandezas cinemáticas.

Suponha que as coordenadas cartesianas das estações sejam $\left(\mathrm{x}_{\mathrm{i}}, \mathrm{y}_{\mathrm{i}}\right), \mathrm{i}=1,2$ e 3 onde as componentes medidas do vento são $\left(\mathrm{u}_{\mathrm{i}}, \mathrm{v}_{\mathrm{i}}\right)$, totalizando, assim 12 quantidades conhecidas. A equação (1) é aplicada sequencialmente a cada estação, usando três pares de pontos: (observação 1 e ponto $\mathrm{O}$ ), (observação 2 , ponto $\mathrm{O}$ ) e (observação 3 , ponto $\mathrm{O}$ ), onde o ponto $\mathrm{O}$ é o centróide do triangulo formados pelas estações e no qual as grandezas cinemáticas são calculadas.

As informações contendo as coordenadas das três estações são expressas por uma matriz $\underline{\underline{E}}$ dada por:

$$
\underline{E}=\left(\begin{array}{cccccc}
1 & 0 & x_{1} & -y_{1} & x_{1} & y_{1} \\
0 & 1 & y_{1} & x_{1} & -y_{1} & x_{1} \\
1 & 0 & x_{2} & -y_{2} & x_{2} & y_{2} \\
0 & 1 & y_{2} & x_{2} & -y_{2} & x_{2} \\
1 & 0 & x_{3} & -y_{3} & x_{3} & y_{3} \\
0 & 1 & y_{3} & x_{3} & -y_{3} & x_{3}
\end{array}\right)
$$

notando-se que a não colinearidade das estações garante que E seja não singular (isto é, seu determinante é não nulo, o que garante sua inversibilidade). As informações dos ventos observados são colocadas na matriz coluna:

$\underline{\mathrm{U}} \equiv\left(\mathrm{u}_{1}, \mathrm{v}_{1}, \mathrm{u}_{2}, \mathrm{v}_{2}, \mathrm{u}_{3}, \mathrm{v}_{3}\right)^{\mathrm{T}}$,

onde o sobre-índice $\mathrm{T}$ designa a transposta (matriz coluna) de $\underline{\mathrm{U}}$. (Isso é feito simplesmente por questão de evitar espaços excessivamente vazios na forma impressa.) Notar que a matriz definida acima é a transposta da correspondente matriz dada em Bluestein (1992), o que consiste em um erro tipográfico nesse livro. Finalmente as incógnitas encontram-se na matriz coluna:

$\underline{\mathrm{S}}=\left(\mathrm{u}_{\mathrm{o}}, \mathrm{v}_{\mathrm{o}}, \cdot, \mathrm{q}, \mathrm{D}_{1}, \mathrm{D}_{2}\right)^{\mathrm{T}}$.

Desse modo, tem-se

$\underline{\mathrm{U}}=\underline{\underline{\mathrm{E}}} \underline{\mathrm{S}}$,

que pode ser invertida resultando a solução

$\underline{\underline{E}}^{-1} \underline{\mathrm{U}}=\underline{\underline{E}}^{-1} \underline{\underline{E}} \underline{\mathrm{S}}=\underline{\underline{\mathrm{I}}} \underline{\mathrm{S}}=\underline{\mathrm{S}}$.

\subsubsection{Método da Integral}

Ao contrário do método matricial, o método da integral fornece separadamente os valores das grandezas a partir do cálculo de integrais de linha apropriadas.

Usando a notação alternativa para vetores onde $\mathbf{V}=(\mathrm{u}, \mathrm{v})$, pode-se escrever de forma compacta as expressões envolvendo integrais de linhas que definem as quatro grandezas cinemáticas: $\delta, \zeta, \mathrm{D}_{1}$ e $\mathrm{D}_{2}$, usando-se o mesmo programa do Mathematica . Essas expressões são agrupadas em:

$\left[\begin{array}{c}\cdot \\ \mathrm{D}_{1} \\ \mathrm{D}_{2}\end{array}\right]=\underset{\mathrm{S} \rightarrow 0}{\operatorname{Lim}} \frac{1}{\mathrm{~S}} \oint\left[\begin{array}{c}(\mathrm{u}, \mathrm{v}) \\ (\mathrm{u}, \mathrm{v}) \\ (\mathrm{u},-\mathrm{v}) \\ (\mathrm{v}, \mathrm{u})\end{array}\right] \bullet\left[\begin{array}{c}\breve{\mathbf{n}} \\ \breve{\mathbf{t}} \\ \breve{\mathbf{n}} \\ \breve{\mathbf{t}}\end{array}\right] \mathrm{ds}$

onde S é área elementar compreendida por um circuito (caminho fechado, no caso um triângulo) e $\hat{\mathbf{n}}$ e $\hat{\mathbf{t}}$ os vetores unitários normal (para fora) e tangente à curva e ds um elemento infinitesimal de distância na direção do versor indicado. Para os casos da divergência e vorticidade, as definições acima estão relacionadas aos teoremas fundamentais do Cálculo Vetorial, a saber, o Teorema de Gauss e o Teorema de Stokes, respectivamente e, são consideradas definições formais dessas grandezas. A generalização da integral para incluir os dois termos de deformação é simplesmente um artifício matemático. A integral de linha de $\mathrm{f}(\mathrm{x}, \mathrm{y})$ - dada pelos produtos escalares da equação (7) ao longo da curva (fechada ou não), a saber:

$\int_{C} f(x, y) d s=\operatorname{Lim}_{K \rightarrow \infty} \sum_{k=1}^{K} f\left(x_{k}, y_{k}\right) \quad s_{k}$,

onde $c$ indica o caminho da integração. 
O algoritmo DIVINT ${ }^{2}$ executa o cálculo das integrais de linha dadas na equação (7) usando a versão 6 do Mathematica. Deve-se notar que não são fornecidos os valores exatos pois não é incluída a passagem do limite da área tendendo para zero (operação impossível no caso de dados reais). Menciona-se que o algoritmo acima, no caso presente de um circuito triangular, as componentes normal e tangencial do vento variam linearmente ao longo dos lados do triângulo.

\subsubsection{Método dos Mínimos Quadrados}

Por esse método procura-se uma superfície ótima de interpolação (superfície ou função interpoladora), do tipo polinomial quando $\mathrm{N}$ observações $\mathrm{f}(\mathrm{x}, \mathrm{y})$ e suas coordenadas $(\mathrm{x}, \mathrm{y})$ são conhecidas para inferir as grandezas cinemáticas referentes a um determinado nível horizontal (ou, isobárico).

A melhor estimativa de $\mathrm{f}(\mathrm{x}, \mathrm{y})$ pode ser obtida minimizando o chamado erro médio quadrático, definido por

$$
=\sum_{\mathrm{i}=1}^{\mathrm{N}}\left[\mathrm{f}\left(\mathrm{x}_{\mathrm{i}}, \mathrm{y}_{\mathrm{i}}\right)-\breve{\mathrm{f}}\left(\mathrm{x}_{\mathrm{i}}, \mathrm{y}_{\mathrm{i}}\right)\right]^{2} \text {. }
$$

No presente caso, $\mathrm{N}=3$ e a superfície interpoladora é um plano ${ }^{3}$ dado por

$\mathrm{f}(\mathrm{x}, \mathrm{y})=\mathrm{a}_{\mathrm{o}}+\mathrm{a}_{1} \mathrm{x}+\mathrm{a}_{2} \mathrm{y}$

que, substituído na equação (9) resulta

$$
=\sum_{\mathrm{i}=1}^{\mathrm{N}}\left[\mathrm{a}_{\mathrm{o}}+\mathrm{a}_{1} \mathrm{x}+\mathrm{a}_{2} \mathrm{y}-\breve{\mathrm{f}}\left(\mathrm{x}_{\mathrm{i}}, \mathrm{y}_{\mathrm{i}}\right)\right]^{2} \text {. }
$$

A determinação dos coeficientes do polinômio interpolador, isto é, $\left\{a_{k}, k=0,1,2\right\}$ é feita através da minimização de $\varepsilon$ com respeito a esses coeficientes. Esse procedimento de minimização requer que

$\frac{\partial}{\partial \mathrm{a}_{\mathrm{k}}}=0 \quad$ para $\mathrm{k}=0,1,2$

o que resulta nas seguintes equações normais

$$
\begin{aligned}
& \frac{\partial}{\partial \mathrm{a}_{\mathrm{o}}}=2 \sum_{\mathrm{i}=1}^{3}\left[\mathrm{a}_{\mathrm{o}}+\mathrm{a}_{1} \mathrm{x}_{\mathrm{i}}+\mathrm{a}_{2} \mathrm{y}_{\mathrm{i}}-\breve{\mathrm{f}}\left(\mathrm{x}_{\mathrm{i}}, \mathrm{y}_{\mathrm{i}}\right)\right]=0 \\
& \frac{\partial}{\partial \mathrm{a}_{1}}=2 \sum_{\mathrm{i}=1}^{3}\left[\mathrm{a}_{\mathrm{o}}+\mathrm{a}_{1} \mathrm{x}_{\mathrm{i}}+\mathrm{a}_{2} \mathrm{y}_{\mathrm{i}}-\breve{\mathrm{f}}\left(\mathrm{x}_{\mathrm{i}}, \mathrm{y}_{\mathrm{i}}\right)\right] \mathrm{x}_{\mathrm{i}}=0 \\
& \frac{\partial}{\partial \mathrm{a}_{2}}=2 \sum_{\mathrm{i}=1}^{3}\left[\mathrm{a}_{\mathrm{o}}+\mathrm{a}_{1} \mathrm{x}_{\mathrm{i}}+\mathrm{a}_{2} \mathrm{y}_{\mathrm{i}}-\breve{\mathrm{f}}\left(\mathrm{x}_{\mathrm{i}}, \mathrm{y}_{\mathrm{i}}\right)\right] \mathrm{y}_{\mathrm{i}}=0
\end{aligned}
$$

\footnotetext{
Os programas desenvolvidos no Mathematica, por questão de espaço não foram incluídos no trabalho. No entanto, eles estão disponíveis, bastando contatar o primeiro autor.

3 Representações de maior ordem (ao invés da linear usada acima) poderiam ser usadas se $\mathrm{N}$ fosse suficientemente grande. Por exemplo, para $\mathrm{N} \geq 6$ uma representação quadrática dada por $f(x, y)=a_{0}+a_{1} x+a_{2} x^{2}+a_{3} y+a_{4} y^{2}+a_{5} x y$
}

Portanto, quando aplicado a um campo vetorial (u, v), as duas componentes (zonal e meridional) são separadamente consideradas. Para o caso da componente zonal u, tem-se

$\mathrm{u}(\mathrm{x}, \mathrm{y})=\mathrm{a}_{\mathrm{o}}+\mathrm{a}_{1} \mathrm{x}+\mathrm{a}_{2} \mathrm{y}$

e as respectivas equações normais tornam-se

$$
\begin{aligned}
& 3 \mathrm{a}_{\mathrm{o}}+\mathrm{a}_{1} \sum_{\mathrm{k}=1}^{3} \mathrm{x}_{\mathrm{i}}+\mathrm{a}_{2} \sum_{\mathrm{k}=1}^{3} \mathrm{y}_{\mathrm{i}}=\sum_{\mathrm{k}=1}^{3} \breve{\mathrm{u}}\left(\mathrm{x}_{\mathrm{i}}, \mathrm{y}_{\mathrm{i}}\right) \\
& \mathrm{a}_{\mathrm{o}} \sum_{\mathrm{k}=1}^{3} \mathrm{x}_{\mathrm{i}}+\mathrm{a}_{1} \sum_{\mathrm{k}=1}^{3} \mathrm{x}_{\mathrm{i}}^{2}+\mathrm{a}_{2} \sum_{\mathrm{k}=1}^{3} \mathrm{x}_{\mathrm{i}} \mathrm{y}_{\mathrm{i}}=\sum_{\mathrm{k}=1}^{3} \mathrm{x}_{\mathrm{i}} \breve{\mathrm{u}}\left(\mathrm{x}_{\mathrm{i}}, \mathrm{y}_{\mathrm{i}}\right) \\
& \mathrm{a}_{\mathrm{o}} \sum_{\mathrm{k}=1}^{3} \mathrm{y}_{\mathrm{i}}+\mathrm{a}_{1} \sum_{\mathrm{k}=1}^{3} \mathrm{x}_{\mathrm{i}} \mathrm{y}_{\mathrm{i}}+\mathrm{a}_{2} \sum_{\mathrm{k}=1}^{3} \mathrm{y}_{\mathrm{i}}^{2}=\sum_{\mathrm{k}=1}^{3} \mathrm{y}_{\mathrm{i}} \breve{\mathrm{u}}\left(\mathrm{x}_{\mathrm{i}}, \mathrm{y}_{\mathrm{i}}\right)
\end{aligned}
$$

As equações (15.a, 15.b e 15.c) constituem um sistema simultâneo de equações algébricas (linearmente independentes pois as três estações, por hipótese, não são colineares), cuja solução, por métodos padrões, fornece os valores desejados de $\mathrm{a}_{\mathrm{o}}, \mathrm{a}_{1}$ e $\mathrm{a}_{2}$. O mesmo procedimento é usado para a componente meridional $v$ quando a função interpoladora é

$\mathrm{v}(\mathrm{x}, \mathrm{y})=\mathrm{b}_{\mathrm{o}}+\mathrm{b}_{1} \mathrm{x}+\mathrm{b}_{2} \mathrm{y}$

A velocidade média, a divergência horizontal, a vorticidade relativa e os dois termos de deformação do escoamento relacionam-se com os coeficientes dos polinômios interpoladores através de

$\left(\mathrm{u}_{\mathrm{o}}, \mathrm{v}_{\mathrm{o}}\right)=\left(\mathrm{a}_{\mathrm{o}}, \mathrm{b}_{\mathrm{o}}\right)$

$\delta \zeta=a_{1}+b_{2}$

$\zeta=b_{1}-a_{2}$

$\mathrm{D}_{1}=\mathrm{a}_{1}-\mathrm{b}_{2}$

$\mathrm{D}_{2}=\mathrm{b}_{1}+\mathrm{a}_{2}$

\subsection{Método Cinemático}

O movimento vertical é, através desse método, obtido pela integração vertical da equação da continuidade. Com relação a um sistema de coordenadas $(\mathrm{x}, \mathrm{y}, \mathrm{z}, \mathrm{t})$ onde a coordenada $\mathrm{z}$ é a altura geométrica, a equação da continuidade é para os propósitos desse trabalho usada em sua forma incompressível ${ }^{4}$, dada por

$\cdot=-\frac{\partial \mathrm{w}}{\partial \mathrm{z}}$

que, integrada de $\mathrm{z}=0$, onde $\mathrm{w}=0$ (superfície plana) até um nível arbitrário z, resulta

\footnotetext{
A formulação matemática do problema em coordenadas isobáricas é praticamente idêntica à aqui apresentada, com atenção somente aos limites das integrais com respeito à pressão. Nessas coordenadas a equação da continuidade tem a mesma forma que a equação (22), sem ser necessário introduzir a hipótese de incompressibilidade.
} 
$\mathrm{w}=-\int_{0}^{\mathrm{z}} \cdot \mathrm{dz^{ \prime }}$

No entanto, existem dificuldades de ordem prática por trás dessa equação aparentemente simples. Elas surgem devido ao fato que os dois termos da divergência horizontal $(\delta)$ apresentam uma forte tendência de cancelamento no caso dos movimentos atmosféricos de grande escala e, como conseqüência, pequenos erros observacionais na medição dos ventos podem acarretar erros relativamente grandes no cálculo dessa grandeza cinemática e, portanto de sua integral vertical usando a equação (23). Por exemplo, um erro de $10 \%$ nas componentes do vento pode implicar em erros da ordem de $100 \%$ na divergência calculada (Holton, 1992).

Como em geral, o sinal algébrico de w permanece o mesmo para todos os níveis troposféricos de uma coluna que se estende da superfície (onde $\mathrm{w}=0$, no caso de ausência de topografia) até à tropopausa $(\mathrm{z}=\mathrm{H}$, onde $\mathrm{w}=0$ é ainda uma aproximação razoável, considerando a grande estabilidade estática da estratosfera), conclui-se que a derivada vertical w(z) deve mudar de sinal em algum nível intermediário. Pela equação (22), tem-se nesse nível onde a derivada é nula que $\delta=0$. Esse nível é conhecido como Nível Não Divergente (NND) e, na atmosfera real é encontrado em torno de $550-600 \mathrm{hPa}$ podendo, no entanto, aparecer com alguma multiciplicidade (dois ou três NND) ou mesmo estar completamente ausente (Bluestein, 1992). Essa configuração de distribuição vertical de divergência horizontal em movimentos de grande escala constitui o chamado princípio de compensação de Dynes.

\subsubsection{Correção}

Pela razão mencionada, a divergência calculada a partir de observações do vento geralmente não satisfaz ao princípio de conservação de Dynes e a equação (23) deve ser de algum modo modificada a fim de se introduzir esse melhoramento (mesmo que artificial). Matematicamente, se esse princípio é violado, deve se ter

$$
\int^{\mathrm{H}} \cdot \mathrm{dz} \mathrm{z}^{\prime} \neq 0
$$

onde $\mathrm{H}$ é a altura da tropopausa.

Desse modo, a correção consiste em modificar o campo de divergência, $\delta$, como obtido usando-se qualquer um dos três métodos descritos nas primeiras seções desse capítulo, gerando-se um novo campo corrigido, $\delta_{\text {cor }}$ Esse último é usado no cálculo do movimento vertical. A correção supõe que o erro na divergência seja proporcional à sua magnitude (isto é, valor absoluto), de modo que

${ }_{\text {cor }}=\cdot+|\cdot|$.

A determinação de $\varepsilon$ é feita impondo que
$\int_{\mathrm{o}}^{\mathrm{H}} \cdot{ }_{\mathrm{cor}} \mathrm{dz} \mathrm{z}^{\prime}=0$

isto é, fazendo que o campo corrigido satisfaça ao princípio de Dynes. Assim, integrando a equação (24) de z $=0$ a z $=H$ tem-se

$$
=-\frac{\int_{0}^{\mathrm{H}} \cdot \mathrm{dz}^{\prime}}{\int_{0}^{\mathrm{H}}|\cdot| \mathrm{dz} \mathrm{z}^{\prime}} .
$$

A velocidade vertical $\mathrm{w}$ em um novel arbitrário $\mathrm{z}$ é obtida usando a mesma equação (23), porém $\operatorname{com} \delta=\delta_{\text {cor }}$. O resultado é

$$
\mathrm{w}(\mathrm{z})=-\int_{0}^{\mathrm{z}} \cdot \mathrm{dz} \mathrm{z}^{\prime}+\frac{\int_{0}^{\mathrm{H}} \delta \mathrm{dz}^{\prime}}{\int_{0}^{\mathrm{H}}|\delta| \mathrm{dz} \mathrm{z}^{\prime}} \int_{0}^{\mathrm{z}}|\delta| \mathrm{dz} \mathrm{z}^{\prime} .
$$

As diferentes integrais da equação (28) são calculadas usando-se o algoritmo OMEGA desenvolvido usando o Mathematica, para cada um dos níveis verticais. No tocante aos dados reais, é importante frisar que o método exige que as três radiossondagens atinjam, no mínimo, o nível da tropopausa, para um determinado horário.

\subsection{Dados}

Neste estudo utilizaram-se os dados de radiossondagem do experimento do LBA/TRMM, realizado na Região Amazônica, coletados em uma região de floresta nativa (Reserva Jarú) e em duas regiões desmatadas (Fazenda Nossa Senhora e uma outra operada por uma equipe de cientistas norte-americanos). A distância média entre estes três sítios é da ordem de 100 km e a diferença em altitude é da ordem de $140 \mathrm{~m}$. Somente resultados para o dia 01 de fevereiro de 1999, às 15 HL (hora local) são mostrados nesse trabalho.

\subsection{Tratamento dos Dados Reais}

A altura da tropopausa foi estimada através da plotagem do perfil vertical de temperatura (Figura 1). A tropopausa, assim estimada, foi em torno de $17.000 \mathrm{~m}$, independente da estação, como de se esperar, devido à proximidade das observações.

Uma vez determinada à altura da tropopausa, uma curva interpoladora (spline de grau 2, como gerada pelo comando INTERPOLATION do Mathematica) foi ajustada à série discreta das componentes u e v do vento. Como o número de pontos dessas séries é relativamente grande, não se nota diferenças pronunciadas entre as representações discreta e contínua (Figura 2). O perfil 
interpolado permite que se escolha um intervalo (submúltiplo da altura da tropopausa menos a do nível mais inferior, no caso 17100 - $300 \mathrm{~m}$ ) de amostragem de modo a se obter uma série discreta igualmente espaçada das componentes u e v. Esse procedimento, além de permitir o uso de filtros, viabiliza o cálculo do movimento vertical w por métodos numéricos.

As séries amostradas (no caso a cada $300 \mathrm{~m}$ ) das componentes do vento a partir do perfil contínuo (interpolado) correspondente são, então, usadas como entrada em qualquer um dos programas do Mathematica (lembrando-se que qualquer um dos métodos reproduz os mesmos resultados). Das quatro grandezas cinemáticas somente uma (divergência) é gravada para ser usada no cálculo do movimento vertical nos níveis troposféricos (exceto pelos valores de contorno na superfície e na tropopausa, onde foram feitos nulos). Os cálculos finais envolvem a determinação da divergência que então é corrigida e usada na obtenção final do perfil vertical de w.

\subsection{Filtragem dos Dados}

Como a divergência horizontal do vento é um campo bastante "ruidoso" (isto é, com muitas componentes de pequenas escalas), seu perfil vertical também o é. Um filtro descrito em Shapiro (1970) foi usado para eliminar seletivamente as componentes de menores comprimentos de ondas (componentes com duas vezes o intervalo vertical de amostragem, ou seja $600 \mathrm{~m}$ ) totalmente eliminadas, com as demais componentes pouco ou nada alteradas). Infelizmente, o problema de filtragem cria um outro adicional, ou seja, a perda de termos nas duas extremidades da série. O algoritmo do filtro é dado por:

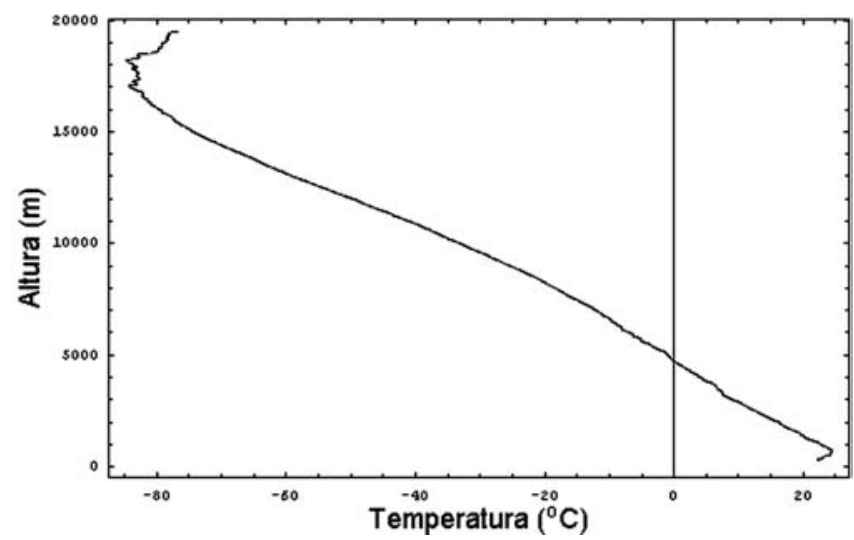

Figura 1 - Perfil vertical da temperatura para a estação de Ouro Preto, no dia 01 de fevereiro de 1999, as 15HL (juntamente com as outras duas estações). A inspeção visual desse perfil foi usada para atribuir aproximadamente a altura da tropopausa $(17.000 \mathrm{~m})$ na região, onde supõe-se que a componente vertical w se anule. Os perfis para as outras duas estações são praticamente os mesmos. $\overline{\delta_{i}}=\frac{1}{256}\left[186 \delta_{i}+56\left(\delta_{i+1}+\delta_{i-1}\right)-28\left(Z_{i+2}+Z_{i-2}\right)+\right.$

$\left.8\left(\mathrm{Z}_{\mathrm{i}+3}+\mathrm{Z}_{\mathrm{i}-3}\right)-\left(\mathrm{Z}_{\mathrm{i}+4}+\mathrm{Z}_{\mathrm{i}-4}\right)\right]$

onde a sobre-barra indica o valor filtrado.

Com esse filtro simétrico envolvendo 8 pontos (quatro de cada lado do ponto em questão) os 4 primeiros e os 4 últimos termo da série são eliminados, ou seja o perfil inicia-se (ao invés de $300 \mathrm{~m}$ ) em $1200 \mathrm{~m}$. Se fosse o caso de maior resolução na camada limite planetária, uma solução paliativa seria aumentar a resolução da amostragem vertical (50 m, por exemplo).

\section{RESULTADOS E DISCUSSÃO}

\subsection{Resultados dos Testes de Validação}

Os métodos desenvolvidos foram validados através de comparações com dados reais (uma única referência) e com valores obtidos através de experimentos com dados teóricos (não mostrados). Bluestein (1992) ilustra o método da integral no cálculo das quatro grandezas cinemáticas com o exemplo de um conjunto de três estações de radiossondagens localizadas nas cidades de Lander (estado de Wyoming), Rapid City (estado de Dakota do Sul) e Denver (estado de Colorado), todas nos Estados Unidos. Os dados referem-se a um único (não mencionado) nível vertical, mas para os propósitos presentes isso é irrelevante. Menciona-se que em Bluestein (1992) contêm erros tipográficos referente aos sinais das componentes do vento em sua Figura 3.42, página 122. A coincidência dos três métodos em reproduzir idênticos resultados (idem quando validados

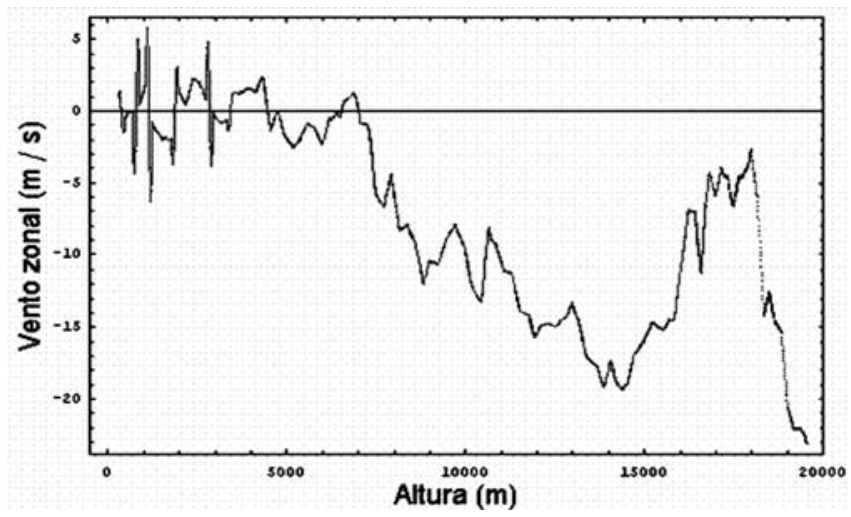

Figura 2 - Perfil vertical da componente zonal do vento para a estação de Ouro Preto, para o dia 01 de fevereiro de 1999, 06 HL. A curva vermelha (contínua) é a curva interpolada que passa pelos pontos discretos originalmente presentes na radiossondagem (não claramente distinguíveis, devido à resolução da figura) a qual é, numa etapa seguinte, amostrada para gerar a série discreta final de 57 pontos (se um intervalo de amostragem de $300 \mathrm{~m}$ for utilizado). 
com dados teóricos) mostra a consistência dos mesmos; afinal usam os mesmos dados de entrada para determinar grandezas em um mesmo ponto.

Observa-se que os resultados coincidentes para os três métodos diferem em torno de $2 \%$ com relação aos de Bluestein (1992), exceto para a divergência (14\%). Essas discrepâncias, no entanto, devem também ser atribuídas a uso de máquinas com diferentes precisões e/ou formato de impressão.

Os três métodos foram também testados com dados teóricos e um circuito quadrado usando os programas DIVINT e VORTIN desenvolvidos no Mathematica, onde a função teste escolhida representa um campo de velocidade hipotético dado por

$\mathrm{u}(\mathrm{x}, \mathrm{y})=\mathrm{x}^{3} \operatorname{sen}\left(\frac{2 \mathrm{y}}{2 \mathrm{c}}\right)$ e $\mathrm{v}(\mathrm{x}, \mathrm{y})=\mathrm{y}^{3} \operatorname{sen}\left(\frac{2 \mathrm{x}}{2 \mathrm{c}}\right)$

Não houve razão especial alguma para essa escolha (exceto talvez pela estética das figuras), mas é possível e facilmente alterar essa função teste.
Os resultados dos testes de comparação são apresentados na Tabela 2, mostrando a precisão do método da integral, comparável aos outros dois (não mostrados).

\subsection{Resultados Usando Dados Reais do LBA}

As figuras 1 e 2 mostram, respectivamente, o perfil vertical da divergência horizontal a partir dos dados originais usando um intervalo de amostragem de 300 m e o mesmo após ser filtrado usando um filtro de Shapiro.

Foram feitos, ainda, um mesmo perfil vertical de divergência após ser corrigida de acordo com a equação (28) e um mesmo perfil vertical de divergência horizontal corrigido e filtrado (figuras não mostradas) usando um filtro de Shapiro (1970) corrigido usando o esquema dado pela equação (25) que impõe o princípio de compensação de Dynes (continuidade de massa na vertical).

Tabela 1 - Resultados dos testes de intercomparação entre os valores obtidos pelos três métodos desenvolvidos e um exemplo com dados reais, já processados.

\begin{tabular}{lcccc}
\hline Grandeza cinemática & Divergência & Vorticidade & Deformação1 & Deformação2 \\
\hline Método da matriz & 0,964 & $-4,892$ & 3,639 & $-4,090$ \\
Método da integral & 0,964 & $-4,892$ & 3,639 & $-4,090$ \\
Método dos mínimos & 0,964 & $-4,892$ & 3,639 & $-4,090$ \\
Valores de referência $^{(1)}$ & 1,1 & $-4,8$ & 3,6 & $-4,2$ \\
\hline \multicolumn{4}{r}{${ }^{(1)}$ Valores que constam no exemplo dado em Bluestein (vol. I,1992). }
\end{tabular}

Tabela 2 - Resultados de testes de comparação no cômputo da vorticidade e divergência.

\begin{tabular}{ccc}
\hline Método & Vorticidade & Divergência \\
\hline Teórico & 3,0000 & $-\pi / 2$ \\
Método da integral & 2,9844 & $-1,5708$ \\
\hline
\end{tabular}

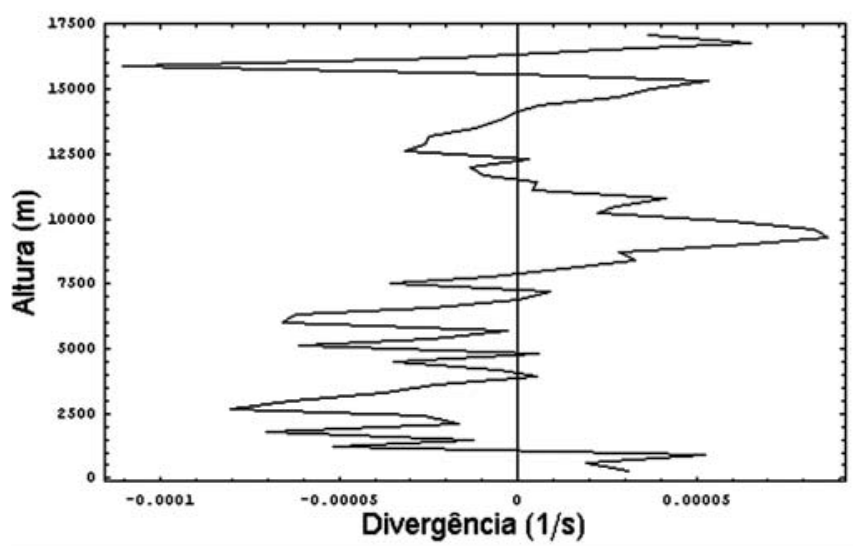

Figura 3 - Perfil vertical de divergência horizontal usando os dados originais para o dia 01 de fevereiro de 1999, as $15 \mathrm{~h}$.

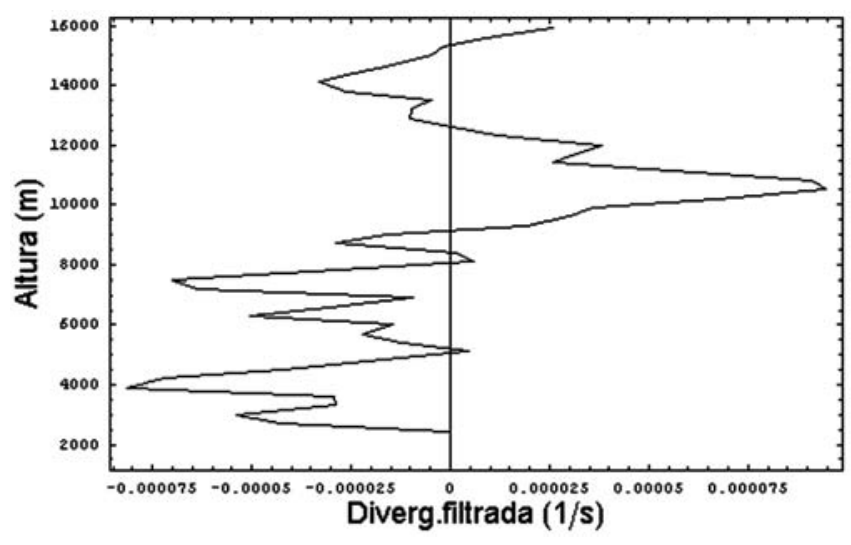

Figura 4 - Perfil vertical de divergência filtrada usando os dados originais para o dia 01 de fevereiro de 1999 , as $15 \mathrm{~h}$. 


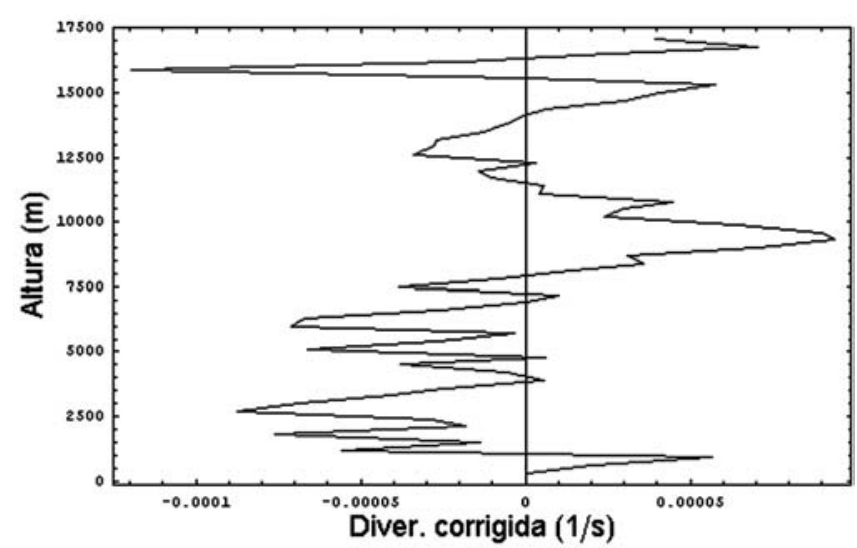

Figura 5 - Perfil vertical de divergência horizontal corrigida (não filtrada) usando os dados originais para o dia 01 de fevereiro de 1999, as $15 \mathrm{~h}$.

As figuras 6 e 7 mostram os perfis verticais de $\mathrm{w}\left(\mathrm{cm} . \mathrm{s}^{-1}\right)$ usando os campos de divergência original e o de divergência corrigida (não filtrado), respectivamente.

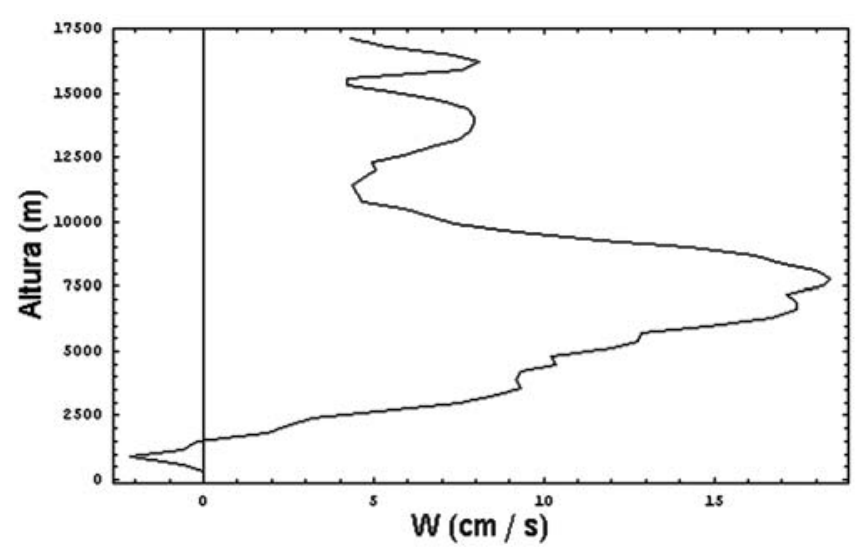

Figura 6 - Perfil vertical da componente $\mathrm{w}\left(\mathrm{cm} \cdot \mathrm{s}^{-1}\right)$ do movimento, a partir da divergência horizontal, para o dia 01 de fevereiro de 1999, as $15 \mathrm{~h}$.

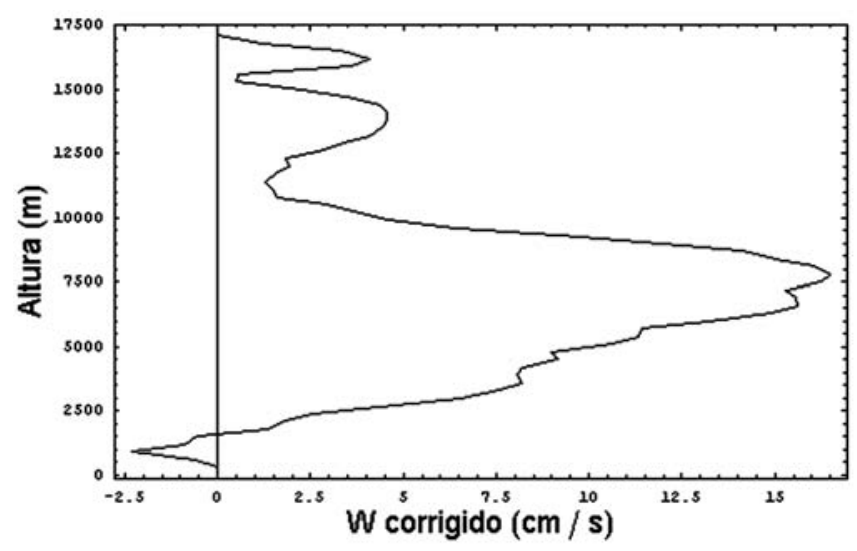

Figura 7 - Perfil vertical da componente $\mathrm{w}\left(\mathrm{cm} . \mathrm{s}^{-1}\right)$ do movimento, a partir da divergência horizontal corrigida, para o dia 01 de fevereiro de 1999 , as $15 \mathrm{~h}$.

\subsection{Análise de Escala}

Nota-se nas figuras 4 e 5 que o máximo do movimento vertical encontra-se a uma altura aproximadamente de $7500 \mathrm{~m}$, portanto bem acima da camada limite atmosférica. Essa distribuição vertical de w é consistente com a estrutura térmica da atmosfera, pois no horário das $15 \mathrm{HL}$ a convecção na região é intensa.

O valor numérico desse máximo é de aproximadamente $15 \mathrm{~cm} . \mathrm{s}^{-1}$. Esse valor também é consistente com o arranjo espacial dos 3 sítios de radiossondagem. De fato, sendo L uma escala de distância horizontal, U uma escala de velocidade horizontal, D uma escala de distância vertical e W uma escala de velocidade vertical, tem-se usando a equação da continuidade que:

$\frac{\mathrm{U}}{\mathrm{L}} \leq \frac{\mathrm{W}}{\mathrm{D}}$

onde a desigualdade aparece devido ao fato dos dois termos da divergência tenderem a se cancelarem nas expressões da divergência horizontal, característica dos fenômenos de grande escala.

Assim, usando as escalas típicas $\mathrm{U} \sim 10 \mathrm{~m} \cdot \mathrm{s}^{-1}$ (de acordo com os dados de vento da radiossodagem), $\mathrm{L} \sim 100 \mathrm{~km}$ (arranjo espacial dos 3 sítios), D $10 \mathrm{~km}$ (altura da tropopausa) segue-se que um limite máximo para $\mathrm{W}$ seria, pela equação (30) da ordem de $100 \mathrm{~cm} / \mathrm{s}$. No entanto, os efeitos de auto-cancelamento dos dois termos na divergência e os advindos da estratificação da atmosfera de grande escala (Holton, 1992, Lemes e Moura, 2002) contribuem para uma redução de uma ordem de magnitude (fator 10), esse valor típico passa a ser da ordem de $10 \mathrm{~cm} / \mathrm{s}$.

\section{CONCLUSÕES}

O presente trabalho teve por objetivo desenvolver, validar e testar um esquema que permita obter o perfil vertical de movimento w a partir de dados de ar superior obtidos em estações não regularmente distribuídas. O esquema foi testado com os dados do Experimento LBA/TRMM e mostrou resultados consistentes que indicam ser promissores em futuros estudos, envolvendo a série completa de 1 mês de dados ( 24 de janeiro a 24 de fevereiro) em problemas de camada limite planetária (convectiva, inclusive) e possíveis interações com a circulação prevalente de grande escala.

\section{REFERÊNCIAS BIBLIOGRÁFICAS}

BERTOLOSSI, R. Um estudo comparativo entre a velocidade vertical e o vetor $Q$ de Hoskins, com alicação em sistemas sinóticos típicos do sul e sudeste do Brasil. 1988, 83f. Dissertação (Mestrado em Meteorologia). Instituto Nacional de Pesquisas Espaciais - INPE, São José dos Campos, 1988. 
BLUESTEIN, H. B. Synoptic-dynamic meteorology in midlatitudes. New York: Oxford University Press, $1^{\text {a }}$ Ed., 1992. $431 \mathrm{p}$.

CHARNEY, J.G. The dynamic of long waves in a baroclinic westerly current. Journal of Meteorology, 4(2), p. 135-163, 1947.

EADY, E.T. Long waves and cyclones waves. Tellus, 1(1), p. 33-52, 1949.

HESS, S. L. Introduction to theoretical meteorology. New York: Henry Holt and Company, 1979. 363 p.

HOLTON, J. R. An introduction to dynamic meteorology. New York: AcademicPress, $3^{\mathrm{a}} \mathrm{Ed}, 1992.511$ p.

HOSKINS, B.J.; DRAHICI, I. and DAVIES, H.C. A new look at the omega equation. Quart. J. Roy. Meteor. Soc., 104, p. 31-38, 1978.

KRISHNAMURTY, T. N. Workbook on numerical weather prediction for the tropics for the training of class $I$ and class II meteorological personnel. World Meteorological Organization, 1986. $669 \mathrm{p}$.
LEMES, M.A.M. e MOURA, A.D. Fundamentos de dinâmica aplicados à meteorologia e oceanografia. Ribeirão Preto: Holos Editora, 2 ${ }^{\text {a }}$ Ed., 2002. 296 p.

O'BRIEN, J.J. A direct numerical solution to a one-dimensional Helmholtz equation. Journal Computational Physics, $\underline{3}$ (3), p. 544-546, 1969.

ORLANSKI, I. A rational subdivision of scales for atmospheric processes. Bull. Amer. Meteor. Soc., 56(5), p. 527-534, 1975.

SHAPIRO, R. Smoothing, Filtering and Boundary Effects. Reviews of Geophysics and Space Physics, v. 8, n. 2, p. 359-387, 1970.

TRENBERTH, K.E. On the Interpretation of the diagnostic quasi geostrophic omega equation. Monthly Weather Review. 106 (1), p. $131-137,1978$.

YANAI, M.; ESBENSEN, S.; CHU, J.H. Determination of bulk properties of tropical cloud clusters from large scale haeat and moisture budgets. J. of Atmos. Sci., v. 30, p. 611 $-627,1973$. 